\title{
Trayectorias de movilidad residencial y cuidado en casa de ancianos pobres urbanos
}

\author{
Residential mobility trajectories \\ and home care of the elderly urban poor
}

\author{
Leticia Robles-Silva*
}

\section{Resumen}

El objetivo es identificar los tipos de movilidad residencial entre los ancianos pobres urbanos con base en los espacios de cuidado en la casa donde residen. Los datos provienen de dos estudios etnográficos en Guadalajara, México, con 40 ancianos pobres urbanos. Se analiza la casa como un espacio de cuidado a partir de las características fisicas y el uso del espacio doméstico, y las relaciones familiares en su interior para identificar los tipos de movilidad residencial. Cuatro trayectorias de movilidad residencial muestran las circunstancias para la inclusión o exclusión del cuidado en casa y la movilidad residencial entre la casa y el asilo.

Palabras clave: movilidad residencial; ancianos; cuidado; pobreza urbana; México.

\begin{abstract}
The objective is to identify the types of residential mobility among the elderly urban poor based on the care spaces in the households where they live. Data are drawn from two ethnographic studies in Guadalajara, Mexico, with elderly urban poor people. The house is analyzed as a care space based on physical characteristics and the use of domestic space and the family relationships within it to identify the types

* Universidad de Guadalajara. Dirección: Juárez 976, Centro, 44100, Guadalajara, Jalisco, México. Correo: leticia.robles.silva@gmail.com ORCID: http://orcid.org/0000-00030119-6300

Nota de la autora: Agradezco al Consejo Nacional de Ciencia y Tecnología (Conacyt) el apoyo para la realización de algunas fases de esta investigación, en particular para el estudio etnográfico realizado en 2007 con 25 familias en el barrio de Oblatos, en Guadalajara (financiamiento 980302020), así como para la realización del estudio en un asilo privado entre 2007 y 2010 (financiamiento 80062).
\end{abstract}


of residential mobility. Four residential mobility paths show the circumstances for the inclusion or exclusion of home care and residential mobility between a person's home and an elderly care facility.

Keywords: residential mobility; elderly; care; urban poverty; Mexico.

\section{Introducción}

Para mí, jen las casas!, como antes, que allí moría uno en la familia, con hermanos. $\mathrm{O}$ a veces se casaban las hijas de uno, y se iba uno con ellas y sus maridos, ahí, a vivir con ellas, como antes. A mí se me hace muy bonito eso.

Esta fue la respuesta de Francisco, un anciano octogenario, a mi pregunta: ¿dónde es mejor vivir cuando se es viejo: en la casa o en un asilo? Francisco había vivido en un barrio popular urbano durante su vida adulta y parte de su vejez, pero después de varios cambios de residencia ingresó a un asilo a causa de su condición de fragilidad. La experiencia de Francisco va en contra de la tendencia en los arreglos residenciales en México, ya que sólo 5\% de los ancianos residen en asilos (United Nations, 2005, p. 52), pues la mayoría de los ancianos corresiden, ya sea con algún hijo adulto (Angel, Vega y López-Ortega, 2017, p. 156; Redondo, Garay y Montes de Oca, 2015, p. 628) o con algún pariente en el caso de los ancianos sin hijos (De Vos, 2014, p. 474).

En México, la familia tiene un papel central en el cuidado de los ancianos; esto es debido a diversas circunstancias, entre ellas, a la escasa protección social (Wong y Palloni, 2009, pp. 238-239), a la carencia de servicios de cuidado de largo plazo en casa (Hopenhayn, Sojo y Mancero, 2012, p. 237) y a las pocas instituciones de servicios de cuidado de largo plazo existentes para los ancianos sin recursos económicos (Angel, Angel, López-Ortega, Gutiérrez y Wallace, 2016, p. 334). En este contexto de limitada protección social por parte del Estado, la familia es el principal recurso de apoyo social y cuidado para los ancianos en México (Pelcastre-Villafuerte, Treviño-Siller, González-Vázquez y Márquez-Serrano, 2011, pp. 462-464; Fuller-Iglesias y Antonucci, 2016, p. 332).

El deterioro en las condiciones de salud del anciano es uno de los motivos más frecuentes para cambiar de residencia (Atkins, 2017, p. 7; Wilmorth, 2010 , p. 874), ya que en condiciones de fragilidad es necesario vivir en un lugar donde se pueda recibir cuidado (Milligan, 2009, p. 67). Por eso, en muchas ocasiones una condición necesaria para el cuidado es la corresidencia del anciano con su familia. Los dos cambios de residencia más comunes 
son: mudarse a casa de algún hijo o residir en una institución de cuidado de largo plazo (van der Pers, Kibele y Mulder, 2017, p. 9). Sin embargo, algunos estudios muestran cómo la movilidad residencial es un proceso gradual en el cual la corresidencia con la familia es una fase intermedia entre residir solo en su casa y el asilo (Hoof, Kort y Waarde, 2009, p. 384; Firbank y Johnson-Lafleur, 2007, p. 188; Hays, 2002, p. 143). Aun así, la casa es la alternativa más aceptada como espacio de cuidado y el lugar principal en el cual los ancianos enfermos o con discapacidad reciben el cuidado informal (Willimans, 2002, pp. 144-147; Bowlby, Gregory y McKie, 1997, pp. 345346); por lo tanto, es común que el cuidado se localice fuertemente en el espacio doméstico (Milligan, 2003, pp. 461-462).

Los estudios en el campo de la geografía del cuidado han mostrado que la casa no siempre es el mejor lugar para ser cuidado; si bien puede ser un lugar adecuado y confortable, también puede ser un espacio de opresión y alineación para los ancianos, los enfermos y las personas con discapacidad (Blunt y Dowling, 2006, p. 10; Gieryn, 2000, pp. 474-475; Brickell, 2012, pp. 232-235). En este sentido, permanecer en casa no necesariamente implica recibir cuidado de calidad. Para entender cómo la casa puede ser o no un espacio idóneo para el cuidado, es necesario considerar varias circunstancias. Por una parte, otorgar cuidado en casa significa cuidar en un espacio planeado para otros propósitos, puesto que los hogares no están diseñados para enfrentar las necesidades de las personas con discapacidad o con enfermedades graves (Imrie, 2004, p. 688; Easterlow y Smith, 2004, p. 1003). La casa es un espacio donde también existen personas y relaciones sociales (Bowlby, Gregory y Mckie, 1997, p. 343); por eso las relaciones de parentesco son otro componente que afecta el cuidado, al normar lo que puede hacerse dentro del hogar, pero también al valorizar ciertas relaciones y marginar a otras (Blunt y Dowling, 2006, p. 22), definiendo a quién se cuida y a quién no (Hareven, 1994, pp. 448-451), lo cual permite entender si los ancianos reciben o no cuidado en casa. Finalmente, algunos autores afirman que recibir cuidado en casa es una circunstancia que afecta la posibilidad de envejecer en casa (Milligan, 2009, pp. 18-20) e influye en el número de veces que un anciano cambia de residencia (Houben, 2000, p. 56). Dado lo anterior, el cuidado en casa es una dimensión a ser considerada para entender la movilidad residencial de los ancianos.

El tema de la movilidad residencial ha sido poco explorado con respecto a los ancianos pobres (Golant, 2008, p. 383). De acuerdo con las evidencias en países desarrollados, y a pesar de las políticas sociales de estos países para otorgar servicios de cuidado en casa o de la existencia de una amplia oferta del mercado, los ancianos pobres experimentan varias condi- 
ciones adversas con respecto al cuidado en casa y la posibilidad de la movilidad residencial. Por una parte, los ancianos pobres tienen pocas opciones para cambiar de residencia y escasamente se mueven (Strohschein, 2012, pp. 741-742; Rosernberg y Everitt, 2001, p. 138); y cuando experimentan movilidad es más probable que ingresen a un asilo (Gaugler, Duval, Anderson y Kane, 2007, pp. 4-9). Asimismo, ellos y sus familias tienen más dificultades para solventar las deficiencias físicas de la vivienda y realizar las adaptaciones de acuerdo con las necesidades de sus residentes ancianos (Rosenberg y Everitt, 2001, p. 140). Según estas evidencias, los ancianos pobres de los países desarrollados estarían experimentando escasa movilidad residencial, permaneciendo en un espacio poco propicio para su cuidado. A pesar de tales evidencias, no sabemos si las mismas circunstancias experimentan los ancianos pobres en países de ingresos medios como, por ejemplo, México.

Entre los pocos estudios sobre la movilidad residencial de los ancianos mexicanos se reporta que una razón para el cambio de residencia es el deterioro en sus condiciones de salud (Capron y González, 2010, p. 72), y dicho deterioro es un factor asociado a corresidir con un hijo (Wong y Palloni, 2009, p. 244; Montes de Oca y Hebrero, 2006, p. 108; Pérez y Brenes, 2006, p. 649). En cambio, los estudios en asentamientos del sector popular urbano reportan que los ancianos propietarios de casas de autoconstrucción casi nunca experimentan movilidad residencial (Ward, 2012, p. 1496; Ward, Jiménez, Huerta, Grajeda y Velázquez, 2011, pp. 471-473; Padilla, 2012, pp. 170-235; Gilbert, 1999, p. 1080). De acuerdo con lo anterior, los hallazgos evidencian un conocimiento fragmentado de la movilidad residencial entre los ancianos pobres en México y escasa atención a los tipos de movilidad residencial en relación con el cuidado en casa o institucional. En este sentido, el objetivo de este trabajo es identificar los tipos de movilidad residencial entre los espacios de cuidado en casa donde residen los ancianos dependientes en el contexto de pobreza urbana.

\section{Estrategia metodológica}

El acercamiento metodológico utiliza la triangulación de datos consistente en analizar material de campo proveniente de diferentes espacios, pero relativos al mismo fenómeno bajo estudio (Denzin, 1997, p. 11); para ello utilizo el material de campo de dos etnografías realizadas en contextos de pobreza urbana en Guadalajara, México. Ambos estudios contienen información acerca de los cambios de residencia y los diferentes tipos de arre- 
glos residenciales experimentados por ancianos, quienes residían en barrios consolidados del sector popular urbano. Este tipo de barrios formaban parte del área marginal de Guadalajara en las décadas de 1950 y 1960, pero con el crecimiento urbano y la dotación de servicios, pasaron a formar parte del anillo intermedio de la ciudad, siendo hoy día barrios populares consolida$\operatorname{dos}^{1}$ (Jiménez, 2015, pp. 9-14).

\section{Lugar y participantes}

La primera etnografía fue realizada con 25 familias en el barrio de Oblatos en Guadalajara (Robles-Silva, 2007, pp. 55-79). El barrio fue fundado en la década de 1960 y para el momento del trabajo de campo era un barrio popular urbano consolidado; esto es, con una pobreza relativa, alta densidad habitacional, casas de autoconstrucción terminadas y con mejoras en sus aspectos arquitectónicos, con calles pavimentadas, dotación de infraestructura y todos los servicios públicos. El segundo estudio lo llevé a cabo entre 2007 y 2010, en un asilo privado, fundado en 1994, dirigido a prestar servicios de cuidado de largo plazo tanto a ancianos pobres como a ancianos desamparados o sin familia. Las familias pagaban 3500 pesos mensualmente, pero debían proporcionar por separado los medicamentos y los pañales. Sin embargo, era frecuente que las familias se atrasaran en el pago de las cuotas o solicitaran la condonación parcial o total de las mismas; por ello, la administración del asilo enfrentaba dificultades económicas para su operación. La capacidad del asilo era para 29 ancianos, pero residieron 47 durante el periodo del trabajo de campo. En ambos sitios se realizaron entrevistas a profundidad con los ancianos y sus familias, además de observación participante; en el asilo también revisé los expedientes de los ancianos y realicé entrevistas semiestructuradas con dos de las trabajadoras sociales y tres grupos focales con las empleadas.

De ambos estudios seleccioné a los ancianos con discapacidad severa, quienes murieron durante el trabajo de campo o eran ancianos muy viejos; y de los residentes del asilo, elegí a quienes antes de su ingreso residían en un barrio popular de Guadalajara.

${ }^{1}$ Los barrios populares urbanos consolidados fueron en su origen asentamientos de los pobres en las periferias de las ciudades mexicanas, los cuales dos o tres décadas después pasaron a localizarse en el anillo intermedio de la ciudad. Dichos barrios exhiben una relativa mejoría en la infraestructura urbana, pero conservan algunos rasgos de pobreza como son: bajos ingresos, inserción al sector informal y falta de acceso a la seguridad social (Ben Amor, Mathieu y Silva, 1991, pp. 236-240; Gilbert, 2011, pp. 80-81). 
Los casos analizados fueron 18 ancianos del trabajo de campo en el barrio y 22 residentes del asilo. La mitad eran hombres y la otra mitad mujeres, con edades entre 73 y 94 años. La mayoría de los residentes del barrio estaban casados, a diferencia de los ancianos del asilo, quienes eran viudos, divorciados o solteros. Todos los ancianos residentes del barrio tenían hijos vivos, 7.4 hijos en promedio, y 16 de los 22 ancianos del asilo también tenían hijos, pero menos: 4.2 hijos en promedio. El único bien en propiedad era su casa; todos los ancianos del barrio, excepto dos, eran propietarios de sus casas; en cambio, sólo nueve de los ancianos del asilo eran propietarios y 13 vivían en casas rentadas o en una casa propiedad de alguno de sus hijos. Su principal fuente de ingresos era el apoyo económico de sus hijos o familiares, y solamente siete del total de ancianos recibían una pensión por jubilación, de alrededor de 2500 pesos mensuales. Todos los ancianos eran dependientes, padecían enfermedades crónicas -como diabetes, hipertensión, artritis, enfermedades cardíacas-, requerían ayuda con las actividades personales e instrumentales de la vida diaria y estaban confinados en sus casas. Además, diez de ellos sufrían de discapacidad motriz severa, por ende, usaban andadera o silla de ruedas para movilizarse, y dos eran ciegos. Por dichas condiciones todos necesitaban de cuidado.

\section{Análisis de los datos}

En la primera etapa analicé la carrera residencial de cada anciano graficando los sitios donde había residido a lo largo del tiempo (Blunt y Dowling, 2006, p. 39); para efectos de este análisis, el punto de partida fue el lugar de residencia durante la vida adulta, registrando cada cambio de residencia durante la vejez, incluyendo el lugar de origen y de destino. En la segunda fase analicé cada lugar de residencia en términos de espacio de cuidado como una estrategia analítica para entender cómo la espacialidad influye en la organización del cuidado informal, cuya propuesta es analizar la casa como un espacio de cuidado, para lo cual hay que entenderla en términos de casa y no solamente en su sentido restringido de vivienda. Esto significa incluir no sólo sus aspectos físicos arquitectónicos, sino también las actividades, los objetos, las relaciones interpersonales, la funcionalidad, el espacio doméstico, los significados, entre otros, existentes en su interior (Bowlby, 2012, pp. 2110-2113). De dichas dimensiones se seleccionaron tres vinculadas con la movilidad residencial: las características físicas de la vivienda, la organización y el uso del espacio doméstico, y el tipo de relación de cuidados existentes en el interior del hogar. La interrelación de estas tres 
dimensiones dentro de la casa fue la base para analizar la inclusión o exclusión del cuidado en casa.

El análisis de las características físicas de la vivienda incluía su tipo, tamaño y propiedad, por ser características que afectan la organización del cuidado (Perkins y Thorns, 1999, pp. 128-131; Houben y van der Vordf, 1993, pp. 303-313). El tamaño de la vivienda fue medido con las herramientas Street View y la regla de Google Earth, calculando en metros cuadrados su dimensión y clasificando las viviendas en pequeñas (100 $\mathrm{m}^{2} \mathrm{o}$ menos), medianas (101- $\left.199 \mathrm{~m}^{2}\right)$ y grandes $\left(200 \mathrm{~m}^{2}\right.$ o más), de acuerdo con la clasificación del mercado inmobiliario mexicano (Bredenoord y Verkoren, 2010, p. 364). El tipo de vivienda fue clasificada en: casa de autoconstrucción, ${ }^{2}$ casa terminada, ${ }^{3}$ o departamento. La propiedad fue clasificada según si el anciano era el propietario, o algún hijo, o si era rentada.

El análisis del uso del espacio doméstico se basó en el concepto de "cuarto para el enfermo" (Rubinstein, 1990, pp. 38-43), un acercamiento que analiza las adaptaciones a la casa en función del uso del espacio doméstico. Esto significa que las familias asignan una habitación para el anciano, y el espacio doméstico y su mobiliario se organiza de tal manera que permita el otorgamiento del cuidado. Tres características fueron analizadas para entender cómo el espacio de la casa era adaptado y negociado para dar cabida al cuidado: 1) si el anciano disponía de una recámara propia, ya que dicha habitación es por lo general donde el cuidado se otorga y se acomoda la parafernalia al respecto (Robles-Silva, 2007, pp. 245-249); 2) si los reacomodos o adaptaciones del espacio doméstico generaban tensión entre los habitantes del hogar; 3) si algún miembro de la familia era desplazado de su recámara para dejársela al anciano.

El análisis de la relación de cuidado incluía la relación de parentesco entre el cuidador y el anciano; si el cuidador asumió el rol gradual o súbitamente; la corresidencia o no entre el cuidador y el anciano; y el número de ancianos residentes en la unidad doméstica con necesidades de cuidado.

${ }^{2}$ En México, la edificación de las viviendas bajo el esquema de autoconstrucción fue la opción para los pobres urbanos, quienes no tuvieron acceso a préstamos de vivienda durante las décadas de 1960 y 1970 (Velázquez, 2015, pp. 525-526). La vivienda de autoconstrucción es una casa unifamiliar con varias habitaciones cuya edificación tarda entre 5 y 15 años. A diferencia de otros países de América Latina, en México los terrenos para la autoconstrucción son grandes, entre $120 \mathrm{~m}^{2}$ y $200 \mathrm{~m}^{2}$ (CIDOC, 2012, p. 137; Gilbert y Ward, 1982, pp. 132-136).

${ }^{3}$ Desde la década de 1990, la mayoría de las opciones de vivienda para los pobres urbanos fue la casa de interés social terminada: viviendas uniformes construidas por desarrollos inmobiliarios. La vivienda es una casa unifamiliar de pequeñas dimensiones de $100 \mathrm{~m}^{2}$ o menos, con 2.1 habitaciones y en dos pisos, la cual no puede ser modificada o expandida a un tercer piso ni añadir habitaciones extras (Bredenoord y Verkoren, 2010, p. 363). De aquí en delante me referiré a este tipo de vivienda como casa terminada. 
La fase final del análisis se basó en el concepto de trayectoria: un proceso social estructurado por una cadena de eventos que no pueden evitarse sin altos costos, con constantes cambios y acciones reactivas o intencionales (Riemann y Shütze, 1991, pp. 336-337). Este acercamiento me permitió analizar diacrónicamente cada cambio de residencia e identificar si el espacio incluía o excluía el cuidado. Cuatro trayectorias de movilidad residencial fueron construidas a partir de conjugar las características de la vivienda, el uso del espacio doméstico y el tipo de relación de cuidado. Para ilustrar estas trayectorias se utilizó la descripción etnográfica como un recurso descriptivo (Ely, Vinz, Downing y Anzul, 1997, pp. 78-93). La narración se basa en los ancianos, quienes mejor ilustran el tipo de movilidad residencial y los cambios de residencia respecto al espacio de cuidado en la trayectoria correspondiente.

\section{Resultados}

El cuidado en casa es el tipo de cuidado central de las cuatro trayectorias, aunque solamente tres de ellas incluyen la movilidad residencial, y cuyos cambios fueron del tipo de movilidad forzada. Esto significa que los cambios de residencia fueron una estrategia para enfrentar una necesidad de cuidado impostergable, obligando al anciano a cambiar de residencia con el fin de recibir dicho cuidado en un espacio valorado como más adecuado en comparación al precedente.

\section{Trayectoria sin movilidad residencial para el cuidado en casa}

Todos los ancianos del barrio permanecieron en la misma casa durante toda su vejez (véase el Esquema 1). Los ancianos eran propietarios de casas grandes de autoconstrucción de $200 \mathrm{~m}^{2}$ con múltiples habitaciones, en donde vivían desde su vida adulta. El cuidado fue incluido en la casa a través de una negociación del uso del espacio doméstico y la disponibilidad permanente de una hija cuidadora.

Los ancianos tenían su propia recámara, no sólo en la que dormían, sino también donde la mayoría de las acciones de cuidado eran realizadas; el mobiliario fue reacomodado para ofrecer un espacio seguro para el anciano, y estas adaptaciones no produjeron tensiones al conservar cada uno de los integrantes su propio espacio y rutinas. 


\section{Esquema 1}

Trayectorias de movilidad residencial

\begin{tabular}{c}
\hline Trayectoria sin movilidad residencial \\
\hline Casa del anciano $\rightarrow \rightarrow \rightarrow \rightarrow$ Muerte \\
Trayectoria de ida y vuelta al cuidado en casa \\
Trayectoria de cuidado transitorio en casa \\
\hline
\end{tabular}

Un solo cambio de residencia

Casa anciano varón $\rightarrow$ Casa hija cuidadora $\rightarrow$ Asilo

Casa anciana $\rightarrow$ Casa anciana. Corresidencia hijo varón $\rightarrow$ Asilo

Varios cambios de residencia

Casa anciano $\rightarrow$ Casa cuidadora $1 \rightarrow$ Casa cuidadora $2 \rightarrow$ Asilo

Trayectoria de cuidado en casa a la distancia

Casa anciano $\rightarrow$ Casa anciano. Ayuda de vecina $\rightarrow$ Asilo

Fuente: Elaboración propia.

Juan, de 75 años, de oficio mecánico, y su esposa Nicolasa, de 73 años, eran propietarios de una casa de $200 \mathrm{~m}^{2}$ donde vivieron por 38 años junto con sus 13 hijos. Poseían una casa grande que les permitió realizar varios arreglos en el uso del espacio doméstico para cuidar primero a Juan y después a su esposa Nicolasa, entre otros; ambos permanecieron en su propia recámara. Otra condición favorable fue la corresidencia con una hija. El retorno o permanencia de hijas casadas, divorciadas o solteras en la casa paterna es una práctica común entre las familias pobres en México (Ward, Jiménez, Grajeda y Velázquez, 2011, p. 471; Varley, 1993, p. 25; United 
Nations, 2005, p. 22). Esta corresidencia con una hija es posible en casas de autoconstrucción, ya que cuentan con habitaciones desocupadas una vez que los hijos se casan y se establecen en una residencia separada. En la casa de Juan y Nicolasa vivía una hija casada con su esposo y tres hijos adolescentes, pues la casa contaba con siete recámaras.

La corresidencia con una hija se acompaña asimismo de un ciclo de reciprocidad. Esta hija casada o divorciada recibe ayuda de sus padres durante crisis familiares o financieras, y después, la hija cuida de sus padres ancianos. En el caso de una hija soltera, ella se convierte en la cuidadora, al ser quien permanece residiendo con ellos. En cualquiera de ambas situaciones, la corresidencia es previa al envejecimiento de los padres, permitiendo asumir progresivamente el rol de cuidadora en la medida en que aumenta la dependencia de sus padres. Otra característica es que la hija asume el cuidado de un anciano a la vez. En el caso de las parejas de ancianos, la hija cuida primero de su padre y después de su madre, o sólo de la madre cuando ésta es viuda desde la edad adulta. La hija de Juan y Nicolasa regresó a la casa paterna porque los ingresos de ella y su esposo eran insuficientes para vivir independientemente; la corresidencia le permitió a la hija no pagar una renta y compartir los gastos del hogar. Al envejecer sus padres, ella cuidó primero a su padre, quien sufría de insuficiencia renal y usaba silla de ruedas al final de su vida, y después a su madre, quien padecía diabetes y enfermedad coronaria.

Este tipo de arreglo residencial de familia extensa también le permite a la hija dedicarse de tiempo completo al cuidado. Aunque los padres ancianos reciben apoyo económico de sus otros hijos, el ingreso principal de la unidad doméstica proviene del yerno y de los nietos. Esto permite a la hija cuidadora trabajar por periodos cortos de tiempo y dedicarse a cuidar a sus padres ancianos. La hija de Juan y Nicolasa solamente trabajaba cuando el ingreso del hogar disminuía, dedicándose al cuidado de sus padres ancianos la mayor parte del tiempo.

La combinación de una casa grande de autoconstrucción con varias habitaciones y una hija cuidadora que correside con los padres desde antes de su envejecimiento es la condición para la inclusión del cuidado en casa sin movilidad residencial. Esas fueron las condiciones de Juan y Nicolasa, ser cuidados en casa hasta su muerte. Pero si no hay una hija cuidadora en casa, aun cuando la casa sea grande, ésta se convierte en un espacio de exclusión del cuidado que se acompaña de movilidad residencial.

Ana, una viuda octogenaria, sin pensión alguna porque su esposo era albañil por cuenta propia, recibía transferencias económicas de sus hijos; ella era propietaria de una casa de autoconstrucción de $200 \mathrm{~m}^{2}$ donde co- 
rresidía una hija casada, quien permaneció ahí después de casarse, con su esposo y dos hijas jóvenes, viviendo en el segundo piso de la casa. La hija de Ana cuidó primero a su padre y después a su madre. Las condiciones para el cuidado en casa de Ana eran ideales, pero su hija murió a causa de un cáncer. Las nietas de Ana asumieron la responsabilidad de cuidar a su abuela cuando murió su madre, pero las circunstancias eran adversas. Ambas nietas trabajaban, por eso acomodaron sus horarios de tal manera que una de ellas siempre estuviera con su abuela, ya que Ana estaba confinada a una silla de ruedas.

La relación de cuidado originó tensión y conflictos entre ambas generaciones. Las nietas enfrentaban las cargas del cuidado y del trabajo al mismo tiempo y, a pesar de sus esfuerzos, Ana se quejaba reiteradamente de no ser cuidada por sus nietas con la misma calidad como lo hacía su hija. Las nietas no podían renunciar a su trabajo porque era un complemento importante del ingreso de la unidad doméstica, ya que las percepciones del padre eran inestables. Después de un año, las nietas se decidieron por el cuidado institucional. Ana experimentó una sola movilidad forzada a un asilo después de vivir 50 años en su casa. Ella vivió en el asilo por cinco años hasta su muerte, a los 83 años. Este cambio de residencia de la casa al asilo muestra cómo el espacio de inclusión del cuidado en casa requiere de ambos componentes: un lugar y una relación de cuidado con una hija de tiempo completo.

\section{Trayectoria residencial de ida y vuelta al cuidado en casa}

Esta trayectoria involucra varios cambios de residencia y combina el cuidado en casa con el cuidado en el asilo (véase el Esquema 1). Los ancianos eran cuatro hombres y dos mujeres, viudos o solteros, quienes vivían solos desde antes de envejecer en una casa de su propiedad o de algún hijo. Todas las viviendas eran casas terminadas o apartamentos de $100 \mathrm{~m}^{2}$. En el caso de quienes eran solteros, no tenían hijos y eran apoyados económicamente por sus hermanos o sobrinos; los viudos tenían hijos, pero sólo uno o dos vivían en Guadalajara, porque los demás habían migrado a Estados Unidos, razón por la cual recibían remesas.

Aunque los ancianos vivían solos, cultivaron fuertes relaciones con su familia desde antes de envejecer. Los ancianos viudos estaban en estrecho contacto y mantenían relaciones de intercambio mutuo con una hija, y los ancianos solteros desarrollaron relaciones de ayuda mutua con alguna hermana. Este tipo de intercambio intergeneracional es conocido como un sistema de familia extensa modificado; esto es, las generaciones tienen una 
relación estrecha y de reciprocidad, pero los ancianos residen en viviendas separadas de los hijos o de sus familiares (Hareven, 1994, p. 441). Tal forma de intercambio es el sustento del cuidado cuando los ancianos sufren de una dependencia moderada. Una hija asume el rol de cuidadora para los padres viudos y una sobrina para los ancianos solteros. La hermana del anciano no participa en el cuidado porque ella a su vez es una anciana dependiente.

El cuidado en casa es organizado a través de la estrategia de cuasi hogares (Albert, 1990, pp. 23-26); ello significa que la cuidadora inicia con las acciones de cuidado mucho tiempo antes de corresidir con el anciano. Este tipo de arreglo para el cuidado consiste, inicialmente, en proporcionar ayuda cotidianamente en tareas tales como el quehacer doméstico y la preparación de alimentos, todas ellas realizadas en la casa del anciano. Durante esta fase, los ancianos experimentan movilidad residencial de corta duración e intermitente hacia la casa de la cuidadora debido a una condición aguda. Una vez que los ancianos se recuperan, ellos regresan a sus casas y las cuidadoras retornan a su rutina cotidiana de cuidado en la casa del anciano.

Pedro era un anciano de 82 años, de oficio electricista, viudo, recibía remesas de sus cinco hijos migrantes y vivía en un departamento de dos recámaras propiedad de su única hija casada, residente en Guadalajara. Durante 19 años su hija lo visitó una vez a la semana; después incrementó las visitas a tres veces por semana, ya que Pedro comenzó a tener dificultades para realizar las actividades instrumentales de la vida diaria debido a la demencia senil. Asimismo, en varias ocasiones, cuando Pedro tenía un padecimiento agudo, se iba a casa de su hija para ser cuidado. Lo mismo sucedía con Amado, de 73 años, soltero y sin hijos, quien era apoyado económicamente por sus sobrinos, y vivía solo en una casa de su propiedad. Amado tenía una fuerte relación con una hermana viuda, quien vivía a dos cuadras de su casa, por lo que cuando sufrió un accidente vascular cerebral, se fue a vivir a casa de ella por varios meses, y su sobrina se convirtió en su cuidadora. Esta última continuó cuidando a su tío Amado cuando él regresó a su casa. La estrategia de cuasi hogares permitió a la hija o a la sobrina incrementar gradualmente las acciones de cuidado conforme declinaban las condiciones de los ancianos y asumían progresivamente el rol de cuidadoras.

Este arreglo de cuidado en casa permanece hasta que las condiciones del anciano se deterioran tanto que es incapaz de vivir solo, razón por la cual se realiza el primer cambio de residencia forzada a la casa de su cuidadora. La hija de Pedro mantuvo esta estrategia de cuasi hogares por cuatro años, hasta que su padre fue incapaz de vivir solo, no solamente por la necesidad de ayuda en las actividades de la vida diaria, sino porque en varias ocasiones se perdió en la calle. La salud de Amado también se fue deterio- 
rando con el paso del tiempo. A pesar del uso de la andadera, las dificultades de movilidad aumentaron y requirió mayor ayuda, incrementándose sus necesidades de cuidado. Ambos ancianos se fueron a vivir a casa de su cuidadora.

Las hijas y las hermanas eran propietarias de viviendas pequeñas tipo casa terminada de tres recámaras. La hija vivía con su esposo y con dos o tres hijos jóvenes; las hermanas eran viudas, pero aún vivían con ellas uno o dos hijos jóvenes solteros. La solución para proporcionar cuidado fue reorganizar el uso del espacio doméstico. Además de reacomodar el mobiliario y debido a que todas las recámaras estaban ocupadas, la recámara de un hijo, sobrino o sobrina fue asignada como el cuarto del abuelo o del tío o la tía. Esta reorganización del espacio doméstico causó conflictos y tensión en el hogar. La hija de Pedro era dueña de una casa terminada de $105 \mathrm{~m}^{2}$, de dos pisos y tres recámaras; para albergar a su padre destinó la recámara de su hijo mayor, y sus tres hijos debieron compartir la misma recámara. Ella también hizo cambios en el acomodo del mobiliario para brindar un ambiente seguro a su padre. Esta fue la única opción ya que, como ella dijo, su casa era pequeña: "la casa es muy chiquita, realmente chiquita". No podía construir otra recámara al no disponer de más espacio. Los conflictos entre los nietos surgieron rápidamente a causa de compartir una misma habitación, y los cambios en el espacio doméstico fueron causa de tensión y conflicto porque rompieron las rutinas de su esposo e hijos.

En las casas de las sobrinas se dio una circunstancia adversa adicional. La sobrina cuidaba simultáneamente a su tío o tía y a su madre. Cuidar de dos ancianos dependientes en la misma casa, al mismo tiempo, incrementó las cargas del cuidado para dicha sobrina cuidadora.

Este arreglo de corresidencia duró poco tiempo debido a los conflictos causados por la reorganización del espacio de la casa y/o por el incremento en la carga al cuidar simultáneamente a dos ancianos dependientes. Pedro vivió en la casa de su hija por seis meses y Amado en casa de su hermana por tres meses. El segundo cambio de residencia forzada fue hacia el asilo, pero el cuidado en casa continuó a pesar de la institucionalización al utilizarse de nuevo la estrategia de cuasi hogares, pero ahora entre la casa y el asilo.

Ambos espacios de cuidado fueron vinculados de dos distintas maneras. Una, el anciano vivía en el asilo durante la semana e iba a casa de la hija o la sobrina los fines de semana o para las celebraciones familiares. La otra, era vivir en el asilo unos meses y otros meses en la casa de la hija o de la sobrina; esto era por razones financieras: cuando la familia no podía pagar la cuota, el anciano regresaba a casa, y cuando la situación financiera mejoraba, el anciano retornaba al asilo. Esta alternancia entre la casa y el asilo 
tenía dos funciones: una, proporcionar un respiro a la cuidadora; dos, permitir a la cuidadora trabajar mientras el anciano vivía en el asilo. La movilidad permanente entre la casa y el asilo mantenía un cierto nivel de cuidado en casa, a pesar de ser casas pequeñas. Pedro y Amado estaban moviéndose permanentemente entre la casa de su hija o hermana y el asilo.

\section{Trayectoria residencial de cuidado transitorio en casa}

Para seis ancianas y tres ancianos varones, el cuidado en casa fue una solución temporal en su trayectoria hacia el asilo. Aquí se presentaron dos tipos de movilidad residencial: una, en la cual la familia intenta una sola vez brindar cuidado en casa; la otra, cuando la familia realiza varios intentos por cuidar en casa antes de optar por el asilo.

El primer tipo de movilidad residencial con un solo cambio de residencia fue entre los ancianos con hijos, quienes vivían solos desde su edad adulta y percibían una pensión. Aquí se combinan relaciones de parentesco débiles con una vivienda, ya sea adecuada o no para el cuidado, donde la corresidencia es más un imperativo que una solución deseada. Los ancianos vivían solos, sin embargo, cuando su salud se deterioró gravemente y fueron incapaces de vivir independientemente, la solución fue la corresidencia con un hijo, pero quién cambiaba de residencia dependía de si el anciano era un hombre o una mujer (véase el Esquema 1).

Los ancianos varones rentaban casa, por eso se movieron a la casa de una hija, quien era propietaria de una casa terminada pequeña de tres recámaras. El primer cambio al llegar los ancianos era la reasignación de las recámaras: los nietos pasaban a compartir una misma recámara para dejar una habitación libre a su abuelo. Esta reorganización del espacio doméstico en una casa pequeña dio origen a conflictos en la familia. Tal fue la experiencia de Francisco, un hombre octogenario, divorciado, pensionado y de oficio sastre, cuando se fue a vivir a casa de su hija Blanca. Ella vivía con su esposo y tres hijos varones jóvenes en una pequeña casa terminada, de $100 \mathrm{~m}^{2}$, de un solo piso y con tres recámaras. No había más espacio, así que cuando su padre sufrió una fractura de cadera, ella aceptó llevarlo a su casa para cuidarlo por insistencia de una de sus hermanas, a quien le dijo: "Bien, no tengo más recámaras, pero haré espacio para él". Cuando Francisco llegó, sus tres nietos ocuparon una sola habitación para dejar libre una recámara para su abuelo. Este arreglo originó conflictos entre los hermanos. Después de seis meses de conflictos, Blanca reacomodó la sala para convertirla en una habitación para su padre, como la única alternativa 
disponible ante la incapacidad económica de construir una recámara extra en un segundo piso.

Los conflictos familiares resultaron no sólo por vivir en una pequeña casa, sino también porque los padres mantenían una relación distante con sus hijas. Usualmente, estos ancianos varones abandonaron a sus familias durante la edad adulta y tuvieron relaciones esporádicas con sus hijos. Cuando envejecieron y sufrieron de dependencia severa, buscaron el apoyo de sus hijos y alguna de las hijas cumplía con la obligación filial. Como consecuencia, establecían de nuevo una relación con su padre, pero al mismo tiempo una relación de cuidado, después de años de no estar en contacto. La hija de Francisco no veía a su padre desde hacía 40 años. Ahora no sólo ambos vivían juntos, sino también cuidaba de él, una responsabilidad inesperada que no era parte de sus expectativas. Aunado a ello, los nietos opinaban que su abuelo no merecía su ayuda por su irresponsabilidad con su familia en el pasado.

En cambio, la corresidencia con las mujeres ancianas se estableció a partir de que algún hijo varón se fue a vivir con ellas (véase el Esquema 1). Las ancianas eran viudas desde antes de envejecer, recibían una pensión por viudez y eran propietarias de una casa grande por autoconstrucción. Por otra parte, la mayoría de sus hijos migraron a Estados Unidos y enviaban regularmente remesas a su madre. Carmen experimentó este tipo de arreglo residencial a sus 84 años. Ella vivía sola y era propietaria de su casa, recibía una pensión por viudez, así como ayuda económica de sus hijos migrantes, pero solamente uno de sus hijos varones vivía en Guadalajara, el resto radicaba en Estados Unidos. Este hijo estaba casado, vivía en una casa rentada y visitaba a su madre una vez a la semana. Cuando las condiciones de salud de Carmen se deterioraron, su hijo, junto con su familia, la nuera y las tres nietas adolescentes, se fueron a vivir a su casa, al ser grande y contar con cinco recámaras.

En México, la relación entre suegra y nuera es conflictiva cuando ambas viven en la misma casa disputando la autoridad doméstica (Arias, 2009, pp. 239-254; Pauli, 2008, pp. 173-177; Varley, 1993, pp. 22-23); añadiéndose a ello, hay conflictos a causa de la relación de cuidado entre ambas. Al mudarse a casa de la suegra, la nuera asume repentinamente el rol de cuidadora. Este fue el caso de la nuera de Carmen, quien se convirtió en su cuidadora en el momento de cambiarse a su casa. Los conflictos aparecieron a causa del cuidado y también por la reorganización del espacio doméstico para alojar a la familia de su hijo, lo cual fue, a su vez, origen de conflictos entre la nuera y el hijo de Carmen.

Tanto para los ancianos varones como para las ancianas, los conflictos permanecieron por varios meses; por esta razón sus familias cuidadoras de- 
cidieron no continuar con el cuidado en casa. La segunda movilidad forzada se da hacia el asilo y el cuidado en casa es reemplazado completamente por el cuidado institucional. Al final, Francisco y Carmen se mudaron al asilo y nunca más regresaron a su casa.

El segundo tipo de movilidad residencial en esta trayectoria implica varios cambios de residencia forzada antes de que el anciano, hombre o mujer, se muden al asilo (véase el Esquema 1). Las condiciones bajo las cuales las cuidadoras se responsabilizan del cuidado explican por qué, a pesar de localizarse el cuidado en una casa de autoconstrucción, se da la movilidad forzada. Los ancianos eran solteros y sin hijos, recibían apoyo económico de sus hermanos y vivían solos en una casa rentada. Ellos habían vivido solos hasta que sufrieron de una dependencia severa y fueron incapaces de vivir independientemente.

El primer arreglo de corresidencia fue una movilidad forzada hacia la casa de una hermana, quien era propietaria de una casa grande por autoconstrucción, donde vivía con algunos de sus hijos solteros y disponía de recámaras desocupadas. Luz vivía sola desde su juventud, era soltera, no tuvo hijos y fue catequista casi toda su vida; fue hasta la edad de 80 años cuando se fue a vivir a la casa de su hermana mayor, quien destinó una de las recámaras desocupadas para ella. Los otros residentes de la casa eran una sobrina y dos sobrinos, todos solteros; fue la sobrina quien asumió el rol de cuidadora. En pocas familias las relaciones entre tíos y sobrinos se mantienen cercanas y duraderas a lo largo del tiempo (Finch y Mason, 1993, pp. 30-32); esto pasó con Luz y su hermana, quienes se visitaban esporádicamente. Sin embargo, cuando Luz sufrió de un accidente vascular cerebral, su sobrina asumió la responsabilidad de cuidarla debido al rápido y súbito deterioro en su salud, sobre todo al quedar ciega, aun cuando ella ya estaba cuidando de su madre. En estas circunstancias, la sobrina de Luz no sólo cuidaba de su madre, sino también de su tía.

La sobrina no trabajaba porque sus hermanos varones eran los proveedores del hogar, por eso se dedicaba de tiempo completo a cuidar de su madre y también de su tía. Sin embargo, el tiempo y el esfuerzo requeridos para atender las necesidades de dos ancianas dependientes fueron la razón, unos meses después, para decidir cuidar solamente a la madre y delegar el cuidado de la tía a otra hermana o prima.

La segunda movilidad forzada fue hacia otra casa de autoconstrucción propiedad de esta otra sobrina. Aquí, Luz también tuvo su propia recámara, pero las condiciones fueron menos favorables para el cuidado en casa. La segunda cuidadora también asumió inesperadamente el rol de cuidadora, pero a diferencia de la primera, no podía dedicarse de tiempo completo al 
cuidado porque trabajaba fuera del hogar. Aunque la combinación de trabajo remunerado y cuidado implicaba una gran presión y carga para la sobrina, no podía dejar de trabajar porque su salario era el principal ingreso de su hogar.

La segunda sobrina cuidadora de Luz era jefa de hogar porque su esposo la abandonó varios años atrás. La sobrina trabajaba en una fábrica y su salario era el principal ingreso de su hogar, ya que sus hijos adolescentes estudiaban y trabajaban de vez en cuando. Estas condiciones de pobreza tuvieron un impacto negativo en el cuidado en casa para su tía, quien permaneció sólo unos meses. La tercera movilidad forzada fue hacia el asilo. Ni Luz ni los otros ancianos regresaron a casa.

\section{Trayectoria residencial de cuidado en casa a la distancia}

Esta trayectoria la experimentaron una anciana y tres ancianos varones, quienes tuvieron una sola movilidad forzada hacia el asilo y el tipo de cuidado en casa era a distancia (véase el Esquema 1). Los ancianos vivían solos, eran viudos o divorciados desde antes de envejecer, y vivían en una casa rentada pero grande, de entre $150 \mathrm{~m}^{2}$ y $200 \mathrm{~m}^{2}$; el espacio doméstico no fue la razón para la movilidad residencial, sino la ausencia de una cuidadora en la casa del anciano.

Ninguno de los hijos asumió el rol de cuidador porque todos migraron a Estados Unidos. En el caso de los hombres ancianos, además, los lazos con sus hijos eran débiles por el poco contacto con ellos después de abandonar o divorciarse de su esposa. No obstante este distanciamiento, tanto los ancianos varones como las ancianas recibían ayuda económica de sus hijos migrantes para la renta, los gastos del hogar y la atención médica.

Los ancianos vivieron independientes hasta que sufrieron de una discapacidad moderada. En ese momento, los arreglos para el cuidado en casa fueron realizados a distancia, ya que los hijos vivían en Estados Unidos, optando por la ayuda de una vecina, quien asumió un relativo papel de cuidadora. La vecina ayudaba con el quehacer doméstico, la preparación de alimentos y los acompañaba a los servicios médicos. Los hijos continuaron dando apoyo financiero para los otros gastos del hogar y pagaban un salario a la vecina por cuidar de su padre o su madre.

Josefina, de 91 años, era viuda, vivía sola y no percibía ninguna pensión, pero sí remesas de sus dos hijos migrantes, quienes la visitaban una vez al año. Ella vivió de manera independiente hasta que comenzó a tener dificultades para realizar las tareas domésticas y a no poder salir sola fuera 
de casa a causa de una discapacidad moderada por su envejecimiento. Sus hijos le pagaron a una vecina para que le ayudara con el quehacer, la acompañara a sus citas con el médico y en ocasiones le ayudara a bañarse.

Un caso distinto fue el de Raúl, de 78 años, quien estaba divorciado, vivía solo y tenía poco contacto con sus cuatro hijos migrantes. Sin embargo, cuando envejeció y sufrió de una discapacidad moderada a causa de un accidente vascular cerebral, buscó la ayuda de sus hijos, quienes decidieron pagar la renta de la casa y a una vecina para que le hiciera el quehacer y le preparara la comida.

Este arreglo de cuidado en casa funcionó bien hasta que los ancianos alcanzaron un estado avanzado de fragilidad y las necesidades de apoyo se incrementaron. En estas condiciones de dependencia, la vecina fue incapaz de satisfacer todas las necesidades de cuidado, y los ancianos ya no podían vivir solos; en esta circunstancia, los hijos decidieron utilizar el cuidado institucional. La única movilidad forzada fue hacia el asilo.

Tanto Josefina como Raúl recibieron apoyo de su vecina hasta que su ayuda fue insuficiente para satisfacer todas sus necesidades. Josefina nunca aceptó irse a vivir con sus hijos a Estados Unidos; los hijos de Raúl se decidieron por el cuidado en un asilo y no por un arreglo de corresidencia porque su padre tenía poco contacto con ellos después del divorcio. Ninguno de los ancianos con esta trayectoria regresó a su casa.

\section{Discusión}

La casa es un recurso importante en el portafolios de los pobres urbanos para enfrentar las condiciones adversas de la pobreza, ya sea utilizándola para actividades productivas o para buscar ayuda entre quienes la habitan (Moser, 1998, p. 24). Las trayectorias de movilidad residencial aquí analizadas dan cuenta de cómo la casa es precisamente un recurso social que también se moviliza para recibir cuidado. Mis hallazgos profundizan en las condiciones por las cuales las familias se deciden por uno u otro tipo de arreglo residencial, a partir de las condiciones de la casa como un espacio de cuidado. Varios puntos merecen ser destacados referentes a los mecanismos de inclusión y exclusión del cuidado en casa y su relación con la movilidad residencial.

Los estudios sobre la migración durante la vejez ubican este tipo de movilidad residencial asociada a la discapacidad como una movilidad en búsqueda de asistencia; es decir, cambiar de residencia para estar cerca de la familia y en caso de necesidad, recibir apoyo social (Wiseman, 1980, pp. 
149-151; Perry, Andersen y Kaplan, 2014, pp. 76-78), pero los cambios de residencia aquí reportados, vistos en su conjunto, dan cuenta más bien de una "movilidad para el cuidado", la cual significa cambiar de residencia y vivir en un espacio en el que se reciba cuidado en condiciones de dependencia moderada o severa, más ligada a la fragilidad del envejecimiento. El mecanismo disparador de esta "movilidad por cuidado" es cuando el espacio habitado por el anciano se transforma en un espacio desfavorable para el cuidado, y entonces se busca un cambio de residencia, donde el espacio cuente con condiciones más idóneas para el cuidado que el anterior. Es decir, esta movilidad es, sobre todo, un proceso de reevaluaciones periódicas de los arreglos residenciales con el fin de recibir cuidado; de ello deriva la decisión de cambiar de residencia o de permanecer en casa, según si el espacio excluye o incluye el cuidado.

La inmovilidad residencial entre los propietarios de casas por autoconstrucción en barrios populares consolidados en las ciudades mexicanas, es un fenómeno visible en décadas recientes; ello en la medida en que, después de medio siglo de existencia, hay evidencias de que muchos de sus primeros pobladores continúan viviendo ahí. Ya con anterioridad, Gilbert (2011, pp. 84-85) afirmaba que la casa de autoconstrucción fue una opción que les permitió a los pobres urbanos tener inicialmente una casa en propiedad; después, y una vez consolidada la vivienda, se convirtió en un recurso que aseguró un lugar donde vivir durante la vejez. Una ventaja adicional, de acuerdo con mis hallazgos, es la posibilidad de recibir cuidado en casa cuando envejecen. Las condiciones favorables para esta inmovilidad residencial son un arreglo de corresidencia con una hija en una casa grande de autoconstrucción, con quien existe una fuerte relación de reciprocidad a lo largo del tiempo. Estos dos rasgos permiten entender por qué algunos ancianos logran no sólo envejecer en su casa por ser propietarios, sino también por qué el cuidado existe en dicho espacio en el largo plazo.

En cambio, las trayectorias con movilidad residencial evidencian cómo alguna variación en estos recursos explica la exclusión del cuidado. La existencia de una vivienda diferente a la de autoconstrucción, $u$ otro tipo de relación de parentesco, son motivo para un cambio de residencia. Condiciones sociales o culturales subyacen en estos factores que excluyen el cuidado en casa.

Los espacios son habitados por personas cuyas relaciones de parentesco norman los intercambios entre ellos. En el caso del cuidado informal, se afirma que las relaciones familiares son su sustento; pero cuando ampliamos la perspectiva a la relación de parentesco, entendemos cómo algunas relaciones familiares funcionan como mecanismos de exclusión del cuidado 
en casa. Una relación filial no siempre es garantía de cuidado; esto acontece cuando se da un rompimiento en el ciclo de reciprocidad por parte del padre. De acuerdo con las expectativas, cuando el padre se divorcia o abandona a la familia y rompe todo contacto con sus hijos, puede ser una razón suficiente para que los hijos no cuiden de su padre anciano (Robles-Silva y Vázquez-Garnica, 2008, pp. 227-228; Varley y Blasco, 2000, pp. 124-125), siendo ello una condición adversa para el cuidado en casa. Las relaciones con parientes lejanos o políticos, como con sobrinas o nueras, también son condiciones adversas para el cuidado en casa. En estos casos, la explicación es porque el cuidado no es parte ni de las expectativas ni de las normas de reciprocidad entre ellos. Los ancianos sin hijos se enfrentan a una débil expectativa familiar de cuidado de parte de sus sobrinos u otros miembros de la familia (Rubinstein, 1987, p. 11), de ahí que las sobrinas asuman el rol de cuidadoras sin sustento normativo. Pero no es sólo esta circunstancia, sino también terminan inmersas en una situación de "cuidadora sándwich", aunque de una naturaleza distinta al concepto usual de este término, relativo al cuidado simultáneo de dos tipos de dependientes: padres ancianos e hijos adolescentes o niños. No es que las sobrinas cuiden de tíos ancianos e hijos pequeños, sino que son "cuidadoras sándwich" por cuidar al mismo tiempo a dos ancianos con distintos parentescos -padres y tíos-, cuyas expectativas son de distinta fuerza normativa, siendo la obligación filial la más fuerte. Una tensión similar se da en la relación entre nueras y suegros. Si hay una relación de parentesco más incierta con respecto al cuidado es con los suegros, porque no es parte de las obligaciones de una nuera (Merrill, 2007, pp. 83-90), ni tampoco existe un ciclo de reciprocidad lo suficientemente fuerte entre ellos como para crear una expectativa de cuidado (Henz, 2010, p. 151). Así, al no existir un vínculo de reciprocidad, ni normas claras sobre si debe o no cuidar, el cuidado en casa también se mantiene por cortos periodos de tiempo cuando la nuera es la cuidadora.

La política social también es parte de este fenómeno del cuidado en casa. De acuerdo con Rosenberg y Everetti (2001, pp. 134-135), pocos países desarrollados han vinculado explícitamente la política de vivienda con la política de cuidado en casa. En el caso de México, a pesar del indiscutible envejecimiento poblacional, no existe una política social de cuidado de largo plazo (López y Jiménez, 2014, pp. 52-54), ni la política de vivienda incluye acciones para el envejecimiento en casa. A pesar de lo anterior, algunas políticas de vivienda del pasado han tenido efectos positivos en la provisión de cuidado en casa en el largo plazo sin habérselo propuesto, tal es el caso de la casa de autoconstrucción. De acuerdo con Smith (2005, p. 2), la flexibilidad en el uso de los espacios domésticos es una condición ne- 
cesaria para sujetar el cuidado a la casa; precisamente, la vivienda de autoconstrucción proporciona un espacio flexible y adaptable a las necesidades de la familia en sus diferentes etapas del ciclo de vida, siendo el cuidado durante la vejez una de esas etapas. En condiciones de pobreza urbana, las familias enfrentan la incapacidad de modificar o adecuar la vivienda a las necesidades de cuidado de los ancianos, de ahí que habitar una vivienda cuyo tamaño y diseño arquitectónico permitan albergar el cuidado es una ventaja en estas condiciones de vulnerabilidad. Una ventaja que la casa terminada no tiene. El punto es relevante porque el cambio en el tipo de vivienda ofertada a los pobres urbanos, e impulsada por la política social a partir de la década de 1990, que es la casa terminada, comienza a dejar visible sus condiciones adversas para el cuidado. En estas trayectorias, los propietarios de dichas casas eran los hijos de los ancianos; ellos fueron quienes enfrentaron las condiciones inadecuadas de sus viviendas para mantener el cuidado de sus padres ancianos en el largo plazo, tanto por el diseño arquitectónico como por su tamaño. En pocos años, estos mismos hijos y sus coetáneos, el próximo contingente de ancianos y por demás más numeroso, experimentarán las mismas condiciones adversas para su propio envejecimiento y cuidado. Si bien accedieron a viviendas, éstas ofrecen una serie de obstáculos para albergar el cuidado que las casas de autoconstrucción de la generación de sus padres no enfrentaron. Futuras investigaciones tendrán ante sí un amplio campo para indagar las respuestas emprendidas por estos futuros ancianos pobres urbanos ante esta circunstancia adversa de la casa terminada.

Otra característica en las trayectorias aquí analizadas fue la naturaleza reactiva de la movilidad; es decir, los cambios de residencia ocurrían hasta cuando las necesidades de cuidado de los ancianos excedían las capacidades del espacio donde vivían, originando un cambio de residencia abrupto. Los hallazgos aquí descritos coinciden con los reportes de otros estudios, en el sentido de que los ancianos cambian de residencia más de forma reactiva que proactiva, debido a que no planifican cambios de residencia con base en sus necesidades futuras de cuidado de largo plazo (Pope y Kang, 2010, pp. 203-204). Incluso, evitan tomar decisiones o planificar acerca de si permanecen o mejor cambian de casa de acuerdo con sus necesidades de cuidado (Löfqvist, Granbom, Himmelsbach, Iwarsson, Oswaldy y Haak, 2013, pp. 922-923). Antes de atribuir esta movilidad reactiva a las condiciones de pobreza de los ancianos, será necesario indagar más sobre el particular. Algunas prácticas de mejoramiento de la vivienda reportadas hoy día entre los sectores populares, las clases medias bajas y en áreas rurales es la verticalidad, es decir, se aumenta a dos o tres pisos la vivienda como una evi- 
dencia de éxito económico (Inclán, 2013, pp. 224-226; Moctezuma, 2017, pp. 503-505; Muñoz y Sánchez, 2017, pp. 112-123), pero en estas mejoras no se consideran las futuras necesidades de cuidado o del propio envejecimiento, como sería subir y bajar escaleras en la cuarta edad de la vida y en condiciones de fragilidad. Tal vez, antes de emitir conclusiones, sea necesario entender la movilidad residencial reactiva en su contexto cultural y no sólo a partir de las condiciones de pobreza.

Este trabajo intenta ofrecer una perspectiva diferente de la movilidad residencial entre ancianos pobres considerando los espacios de cuidado por los cuales transitan. Sin embargo, habría que considerar algunas limitaciones. Los hallazgos se refieren a una movilidad residencial intraciudad, pero existen otros tipos de movilidad, como la intraestatal o la internacional; ello hace interesante una futura agenda de investigación sobre estos otros tipos de migración, así como incluir estudios en diversos grupos sociales, como serían las clases medias o los pobres rurales, que en su conjunto podrían ampliar el panorama de este fenómeno de la movilidad relacionada con los espacios de cuidado.

Un tema de particular interés es la movilidad internacional, que resulta de particular interés para futuras investigaciones dada la bien conocida migración de México a Estados Unidos y los cambios registrados al respecto en los últimos años. Varios estudios han reportado diferentes flujos de migración de ancianos mexicanos entre ambos países. Uno es el correspondiente a ancianos que migran de México a Estados Unidos, quienes han sido admitidos como padres de ciudadanos norteamericanos. Otro flujo es el de los migrantes que retornan a México cuando envejecen y se jubilan con una pensión (Treas y Batalova, 2009, pp. 373-374). El tercer flujo es el de los migrantes ancianos que retornan a México por motivos de salud (Durand, 2004, p. 112; Mojica, 2016, pp. 93-94; Mestries, 2013, p. 191). Futuras investigaciones podrían analizar estos flujos migratorios y tipo de movilidad residencial para identificar no sólo la movilidad en búsqueda de asistencia, sino también la movilidad para el cuidado, como la aquí documentada.

Por último, el análisis del espacio de cuidado en casa a partir de estos dos recursos sociales, las características de la vivienda y el uso del espacio doméstico, junto con las relaciones de parentesco en su interior, muestran que las respuestas de las familias pobres urbanas en barrios populares consolidados no se orientan hacia una trayectoria única de movilidad residencial, ni es una experiencia uniforme de cambios ni arreglos residenciales. Por el contrario, las familias optan por múltiples rutas y múltiples desenlaces de movilidad a partir de sus recursos y las opciones disponibles, a pesar de pertenecer al mismo grupo social. 


\section{Bibliografía}

Albert, S. (1990). The dependent elderly, home health care and strategies of household adaptation. En J. Gubrium y A. Sankar (coords.), The home care experience. Ethnography and policy (pp. 19-36). Newbury Park, CA: Sage.

Angel, J., Angel, R., López-Ortega, M., Gutiérrez, L. y Wallance, R. (2016). Institutional context of family eldercare in Mexico and the United States. Journal of Cross-Cultural Gerontology, 31(3), 327-336. Recuperado de https://link.springer.com/article/10.1007/s10823-016-9291-3

Angel, J. L., Vega, W. y López-Ortega, M. (2017). Aging in Mexico: Population trends and emerging issues. The Gerontologist, 57(2), 157-162. Recuperado de https://academic.oup.com/gerontologist/ article $/ 57 / 2 / 153 / 2646622$

Arias, P. (2009). Del arraigo a la diáspora. Dilemas de la familia rural. Ciudad de México: Miguel Ángel Porrúa.

Atkins, M. (2017). On the move, or staying put? An analysis of intrametropolitan residential mobility and ageing in place. Population, Space and Place, 24(3). Recuperado de https://doi.org/10.1002/psp.2096

Ben Amor, L., Mathieu, D. y Silva, H. (1991). Trayectorias sociales y acceso a la vivienda en el Valle de Chalco. Revista Mexicana de Sociología, 53(1), 225-258. Recuperado de https://www.jstor.org/ stable/3540835?seq=1\#metadata_info_tab_contents

Blunt, A. y Dowling, R. (2006). Home. Londres, Inglaterra: Routledge.

Bowlby, S. (2012). Recognising the time-space dimensions of care: Caringscapes and carescapes. Environment and Planning A, 44(9), 21012118. Recuperado de https://journals.sagepub.com/doi/pdf/10.1068/ a44492

Bowlby, S., Gregory, S. y McKie, L. (1997). Doing home: Patriarchy, caring, and space. Women's Studies International Forum, 20(3), 343350. Recuperado de https://www.sciencedirect.com/science/article/pii/ S0277539597000186

Bredenoord, J. y Verkoren, O. (2010). Between self-help and international housing: A bird's eye view of Mexico's housing production for low and (lower) middle-income groups. Habitat International, 34(3), 59-365. Recuperado de https://www.sciencedirect.com/science/article/abs/pii/ S0197397509001040

Brickell, K. (2012). Mapping and doing critical geographies of home. Progress in Human Geography, 36(2), 225-244. Recuperado de https:// journals.sagepub.com/doi/10.1177/0309132511418708 
Capron, G. y González, S. (2010). Movilidad residencial de los adultos mayores y trayectorias de vida familiares en la ZMVM. Alteridades, 20(39), 67-78. Recuperado de http://www.scielo.org.mx/scielo. php?script=sci_arttext\&pid=S0188-70172010000100006\&lng=es\& nrm=iso\&tlng=es

Centro de Investigación y Documentación de la Casa (CIDOC). (2012). Current housing situation in Mexico 2012. Ciudad de México: Centro de Investigación y Documentación de la Casa.

De Vos, S. (2014). Biologically childless women $60+$ often live in extended family households in Latin America. Journal of Cross-Cultural Gerontology, 29(4), 467-480. Recuperado de https://www.researchgate.net/ publication/267045382_Biologically_Childless_Women_60_Often_ Live_in_Extended_Family_Households_in_Latin_America

Denzin, $\bar{N}$. (1997). Interpretive ethnography. Ethnographic practices for the 21st century. Thousand Oaks, CA: Sage.

Durand, J. (2004). Ensayo teórico sobre la migración de retorno. El principio del rendimiento decreciente. Cuadernos Geográficos, 35(2), 103-16. Recuperado de http://www.ugr.es/ cuadgeo/docs/articulos/035/035-006. pdf

Easterlow, D. y Smith, S. (2004). Housing for health: Can the market care? Environment and Planning A, 36(6), 999-1017. Recuperado de https:// journals.sagepub.com/doi/pdf/10.1068/a36178

Ely, M., Vinz, R., Downing, M. y Anzul, M. (1997). On writing qualitative research living by words. Londres, Inglaterra: Routledge Falmer.

Finch, J. y Mason, J. (1993). Negotiating family responsibilities. Londres, Inglaterra: Routledge.

Firbank, O. E. y Johnson-Lafleur, J. (2007). Older persons relocating with a family caregiver: Processes, stages, and motives. Journal of Applied Gerontology, 26(2), 182-207. Recuperado de https://journals.sagepub. com/doi/pdf/10.1177/0733464807300224

Fuller-Iglesias, H. y Antonucci, T. (2016). Convoys of social support in Mexico: Examining socio-demographic variation. International Journal of Behavior Development, 40(4), 324-333. Recuperado de https:// journals.sagepub.com/doi/pdf/10.1177/0165025415581028

Gaugler, J., Duval, S., Anderson, K. y Kane, R. (2007). Predicting nursing home admission in the U.S: A meta-analysis. BMC Geriatrics, 7(13), 1-14. Recuperado de https://bmcgeriatr.biomedcentral.com/ articles/10.1186/1471-2318-7-13 
Gieryn, T. (2000). A space for place in sociology. Annual Review of Sociology, 26, 463-496. Recuperado de https://www.annualreviews.org/ doi/10.1146/annurev.soc.26.1.463

Gilbert, A. (1999). A home is for ever? Residential mobility and homeownership in self-help settlements. Environment and Planning A, 31(6), 1073-1091. Recuperado de https://ideas.repec.org/a/pio/envira/ v31y1999i6p1073-1091.html

Gilbert,A.(2011). Ten myths undermining LatinAmerican housing policy.Revista de Ingeniería , 35, 79-87. Recuperado de http:/www.scielo.org.co/ scielo.php?script $=$ sci_abstract\&pid $=$ S0121-49932011000300013\&ln$\mathrm{g}=$ es\&nrm=is.\&tlng=en

Gilbert, A. y Ward, P. (1982). Residential movement among the poor: The constraints on housing choice in Latin American cities. Transactions of the Institute of British Geographers, 7(2), 129-149. Recuperado de https://www.jstor.org/stable/622218?origin=crossref\&seq=1\#metadata info tab_contents

Golant, $\bar{S} .(2008)$. Commentary: Irrational exuberance for the aging in place of vulnerable low-income older homeowners. Journal of Aging and Social Policy, 20(4), 379-397. Recuperado de https://www.tandfonline. com/doi/pdf/10.1080/08959420802131437?needAccess=true

Hareven, T. (1994). Aging and generational relations: A historical and life course perspective. Annual Review of Sociology, 20, 437-61. Recuperado de https://www.annualreviews.org/doi/10.1146/annurev. so. 20.080194 .002253

Hays, J. (2002). Living arrangements and health status in later life: A review of recent literature. Public Health Nursing, 19(2), 136-151. Recuperado de https://onlinelibrary.wiley.com/doi/full/10.1046/ j.1525-1446.2002.00209.x

Henz, U. (2010). Parent care as unpaid family labor: How do spouses share? Journal of Marriage and Family, 72(1), 148-164. Recuperado de https://onlinelibrary.wiley.com/doi/10.1111/j.1741-3737.2009.00689.x

Hoof, van, J., Kort, H. S. M. y Waarde, van, H. (2009). Housing and care for older adults with dementia: A European perspective. Journal of Housing and the Built Environment, 24(3), 369-390. Recuperado de https://research.tue.nl/en/publications/housing-and-care-for-olderadults-with-dementia-a-european-perspe

Hopenhayn, M., Sojo, A. y Mancero, X. (2012). Panorama social de América Latina. Santiago de Chile: United Nations Publications.

Houben, P. (2000). Towards a conceptual framework for ageing in place of frail older adults. European Journal of Social Quality, 2(1), 47- 
65. Recuperado de https://www.berghahnjournals.com/view/journals/ ejsq/2/1/eq020104.xml

Houben, P. y van der Voordt, T. (1993). New combinations of housing and care for the elderly in the Netherlands. Journal of Housing and the Built Environment, 8(3), 301-325. Recuperado de https://link.springer. com/article/10.1007/BF02496601

Imrie, R. (2004). Housing quality, disability and domesticity. Housing Studies, 19(5), 685-690. Recuperado de https://www.tandfonline.com/doi/ abs/10.1080/0267303042000249143

Inclán, M. C. (2013). The Casas GEO movement: An ethnography of a new housing experience in Cuernavaca, Mexico. (Tesis de doctorado, London School of Economics and Political Science, Londres). Recuperado de http://etheses.lse.ac.uk/726/1/Inclan-Valadez_ethnography_housing _ Mexico_public.pdf

Jiménez, J. (2015). Colonias populares y su consolidación. En E. R. Jiménez Huerta y H. Cruz Solís (coords.), Colonias populares consolidadas del área metropolitana de Guadalajara. Restos y oportunidades (pp. 9-22). Guadalajara: Universidad de Guadalajara.

Löfqvist, C., Granbom, M., Himmelsbach, I., Iwarsson, S., Oswald, F. y Haak, M. (2013). Voices on relocation and aging in place in very old age. A complex and ambivalent matter. The Gerontologist, 53(6), 919-927. Recuperado de https://academic.oup.com/gerontologist/ article $/ 53 / 6 / 919 / 635643$

López, M. y Jiménez, J. (2014). Dependencia y cuidados de largo plazo. En L. M. Gutiérrez-Robledo, M. C. García-Peña y J. E. Jiménez (coords.), Envejecimiento y dependencia. Realidades y previsión para los próximos años. Documento de postura (pp. 29-62). Ciudad de México: Academia Nacional de Medicina.

Merrill, D. (2007). Mothers-in-law and daughters-in-law: Understanding the relationship and what makes them friends or foe. Westport, CT: Greenwood.

Mestries, F. (2013). Los migrantes de retorno ante un futuro incierto. Sociológica, 28(78), 171-212. Recuperado de https://www.redalyc.org/ pdf/3050/305026407006.pdf

Milligan, C. (2003). Location or dis-location? Towards a conceptualization of people and place in the care-giving experience. Social and Cultural Geography, 4(4), 455-470. Recuperado de https://www.tandfonline. $\mathrm{com} / \mathrm{doi} / \mathrm{pdf} / 10.1080 / 1464936032000137902$ ?needAccess $=$ true

Milligan, C. (2009). There's no place like home: Place and care in an ageing society. Farnham, Inglaterra: Ashgate. 
Moctezuma, V. (2017). Experiencia y significados simbólicos de los habitantes de conjuntos urbanos de interés social en México: segregación, diferencia y distinción. Estudios Demográficos y Urbanos, 32(3), 487514. Recuperado de https://estudiosdemograficosyurbanos.colmex.mx/ index.php/edu/article/view/1723/1801

Mojica, O. A. (2016). Retornos sin familia: el caso de migrantes jubilados. Culturales, 4(2), 79-101. Recuperado de http://www.scielo.org.mx/ scielo.php?script=sci_arttext\&pid=S1870-11912016000200079

Montes de Oca, V. y Hebrero, M. (2006). Eventos cruciales y ciclos familiares avanzados: el efecto del envejecimiento en los hogares de México. Papeles de Población, 12(50), 97-116. Recuperado de http://www. scielo.org.mx/pdf/pp/v12n50/v12n50a6.pdf

Moser, C. (1998). Reassessing urban poverty reduction strategies: The asset vulnerability framework. World Development, 26(1), 21-45. Recuperado de https://www.sciencedirect.com/science/article/abs/pii/ S0305750X97100158

Muñoz, M. y Sánchez, I. (2017). La evidencia del éxito. Residencias y mausoleos en Santiaguito, Arandas, Jalisco. En P. Arias (coord.), Migrantes exitosos. La franquicia social como modelo de negocios (pp. 99-148). Guadalajara, México: Universidad de Guadalajara.

Padilla, P. (2012). Movilidad residencial en asentamientos de origen irregular en Guadalajara 1970-2009. (Tesis de doctorado, Universidad de Guadalajara, México).

Pauli, J. (2008). A house of one's own: Gender, migration, and residence in rural Mexico. American Ethnologist, 35(1), 171-187. Recuperado de https://anthrosource.onlinelibrary.wiley.com/doi/epdf/10.1111/j.15481425.2008.00012.x

Pelcastre-Villafuerte, B. E., Treviño-Siller, S., González-Vázquez, T. y Márquez-Serrano, M. (2011). Apoyo social y condiciones de vida de adultos mayores que viven en la pobreza urbana en México. Cadernos de Saúde Pública, 27(3), 460-470. Recuperado de http://www.scielo. br/pdf/csp/v27n3/07.pdf

Pérez, J. y Brenes, G. (2006). Una transición en edades avanzadas: cambios en los arreglos residenciales de adultos mayores en siete ciudades latinoamericanas. Estudios Demográficos y Urbanos, 21(3), 625-666. Recuperado de https://estudiosdemograficosyurbanos.colmex.mx/index. php/edu/article/view/1243

Perkins, H. y Thorns, D. (1999). House and home and their interaction with changes in New Zealand's urban system, households and family struc- 
tures. Housing, Theory and Society, 16(3), 124-135. Recuperado de https://www.tandfonline.com/doi/abs/10.1080/14036099950149983

Perry, T., Andersen, T. y Kaplan, D. (2014). Relocation remembered: Perspectives on senior transition in the living environment. The Gerontologist, 54(1), 75-81. Recuperado de https://www.ncbi.nlm.nih.gov/ pubmed/23840021

Pope, N. y Kang, B. (2010). Residential relocation in later life: A comparison of proactive and reactive moves. Journal of Housing for the Elderly, 24(2), 193-207. Recuperado de https://www.tandfonline.com/doi/ abs/10.1080/02763891003757122

Redondo, N., Garay, S. y Montes de Oca, V. (2015). Modalidades de allegamiento residencial en la población adulta mayor argentina y mexicana: determinantes socioeconómicos y diferencias regionales. Estudios Demográficos y Urbanos, 30(3), 597-649. Recuperado de https:// estudiosdemograficosyurbanos.colmex.mx/index.php/edu/article/ view/1495/1724

Riemann, G. y Schütze, F. (1991). Trajectory as a basic theoretical concept for analyzing suffering and disorderly social processes. En D. R. Maines (coord.), Social organization and social process: Essays in honor of Anselm Strauss (pp. 333-358). Nueva York, NY: Aldine de Gruyter.

Robles-Silva, L. (2007). La invisibilidad del cuidado a los enfermos crónicos. Un estudio cualitativo en el Barrio de Oblatos. Guadalajara: Editorial Universitaria.

Robles-Silva, L. y Vázquez-Garnica, K. (2008). El cuidado a los ancianos: las valoraciones en torno al cuidado no familiar. Texto and Contexto Enfermagem, 17(2), 225-231. Recuperado de https://www.redalyc.org/ articulo.oa?id=71417202

Rosenberg, M. y Everitt, J. (2001). Planning for aging populations: Inside or outside the walls. Progress in Planning, 56(3), 119-168. Recuperado de https://www.sciencedirect.com/science/article/pii/S0305900601000149

Rubinstein, R. (1987). Childless elderly: Theoretical perspectives and practical concerns. Journal of Cross-Cultural Gerontology, 2(1), 1-14. Recuperado de https://link.springer.com/article/10.1007/BF00117173

Rubinstein, R. (1990). Culture and disorder in the home care experience: The home as sickroom. En J. Gubrium y A. Sankar. (coords.), The home care experience. Ethnography and policy (pp. 37-58). Newbury Park, CA: Sage.

Smith, S. (2005). States, markets and an ethic of care. Political Geography, 24(1), 1-20. Recuperado de https://www.sciencedirect.com/science/ article/pii/S0962629804001532 
Strohschein, L. (2012). I want to move, but cannot: Characteristics of involuntary stayers and associations with health among Canadian seniors. Journal of Aging and Health, 24(5), 735-751. Recuperado de https:// www.ncbi.nlm.nih.gov/pubmed/22219208

United Nations. (2005). Living arrangements of older persons around the world. Nueva York, NY: United Nations.

Treas, J. y Batalova, J. (2009). Immigrants and aging. En P. Uhlenberg (coord.), International Handbook of Population Aging (pp. 365-394). Dordrecht, Países Bajos: Springer.

van der Pers, M., Kibele, E. U. B. y Mulder, C. (2017). Health and its relationships with residential relocations of older people to institutions versus to independent dwellings. Journal of Population Ageing, 11(4), 329-347. Recuperado de https://doi.org/10.1007/s12062-017-9187-1

Varley, A. (1993). Gender and housing. The provision of accommodation for young adults in three Mexican cities. Habitat International, 17(4), 13-30. Recuperado de https://www.sciencedirect.com/science/article/ pii/0197397593900269

Varley, A. y Blasco, M. (2000). Exiled to the home: Masculinity and ageing in urban Mexico. The European Journal of Development Research, 12(2), 115-138. Recuperado de https://link.springer.com/ article/10.1080\%2F09578810008426768

Ward, P. (2012). A patrimony for the children: Low-income homeownership and housing (im)mobility in Latin American cities. Annals of the Association of American Geographers, 102(6), 1489-1510. Recuperado de https://www.tandfonline.com/doi/abs/10.1080/00045608.2011.628260

Ward, P., Jiménez, E., Grajeda, E. y Velázquez, C. (2011). Self-help housing policies for second generation inheritance and succession of "The house that mum and dad built". Habitat International, 35(3), 467485. Recuperado de https://www.sciencedirect.com/science/article/pii/ S0197397510000883

Williams, A. (2002). Changing geographies of care: Employing the concept of therapeutic landscapes as a framework in examining home space. Social Science and Medicine, 55(1), 141-154. Recuperado de https:// www.ncbi.nlm.nih.gov/pubmed/12137183

Wilmoth, J. M. (2010). Health trajectories among older movers. Journal of Aging and Health, 22(7), 862-881. Recuperado de https://journals. sagepub.com/doi/pdf/10.1177/0898264310375985

Wiseman, R. (1980). Why older people move: Theoretical issues. Research on Aging, 2(2), 141-154. Recuperado de https://journals.sagepub.com/ doi/abs/10.1177/016402758022003 
Wong, R. y Palloni, A. (2009). Aging in Mexico and Latin America. En P. Uhlenberg (coord.), International Handbook of Population Aaging (pp. 231-252), Dordrecht, PaísesBajos: Springer.

\section{Acerca de la autora}

Leticia Robles Silva es doctora en Ciencias Sociales por la Universidad de Guadalajara-CIESAS. Sus intereses de investigación radican en los estudios de familia, el cuidado y el envejecimiento, utilizando metodologías cualitativas, en especial la etnografía. Sus líneas de investigación se centran en los aspectos culturales y morales del cuidado, así como en los aspectos sociales del entorno que influyen en la organización del cuidado dentro de la familia. Es profesora-investigadora en la Universidad de Guadalajara, y actualmente está realizando una investigación sobre el papel de la familia en el cuidado de ancianos que viven solos en una ciudad media de Uruguay. Pertenece al Sistema Nacional de Investigadores. ORCID: http://orcid.org/0000-00030119-6300

Entre sus publicaciones se encuentran:

Robles-Silva, L. (2007). La invisibilidad del cuidado a los enfermos crónicos. Un estudio cualitativo en el barrio de Oblatos. Guadalajara: Universidad de Guadalajara, Centro Universitario de Ciencias de la Salud. Robles-Silva, L. y Rosas-García, M. D. (2014). Herencia y cuidado: transiciones en la obligación filial. Desacatos. Revista de Antropología Social, 45, 99-112. Disponible en http://www.scielo.org.mx/scielo. php?script=sci_arttext\&pid=S1607-050X2014000200009

Recepción: 29 de mayo de 2018.

Aceptación: 27 de septiembre de 2018. 